\title{
Stochastization as a possible cause for fast reconnection during MHD mode activity in the ASDEX Upgrade tokamak
}

\author{
V. Igochine ${ }^{1}$, O. Dumbrajs ${ }^{2,3}$, D. Constantinescu ${ }^{4}$, H. Zohm ${ }^{1}$, G. Zvejnieks ${ }^{3}$ and ASDEX Upgrade Team ${ }^{1}$ \\ ${ }^{1}$ MPI für Plasmaphysik, Euratom-Association, D-85748 Garching, Germany \\ ${ }^{2}$ Helsinki University of Technology, Association Euratom-Tekes, P.O.Box 2200, FIN-02015 HUT, Finland \\ ${ }^{3}$ Institute of Solid State Physics, Association Euratom-University of Latvia, Kengaraga Street 8, LV-1063, Riga, Latvia \\ ${ }^{4}$ Department of Applied Mathematics, University of Craiova, A.I.Cuza Street 13, Craiova 1100, (200585) Romania
}

The role of stochastization of magnetic field lines is analyzed in fast reconnection phenomena occurring in magnetized fusion plasma during various conditions in the ASDEX Upgrade tokamak. The mapping technique is applied to trace the field lines of toroidally confined plasma where perturbation parameters are expressed in terms of experimental perturbation amplitudes determined from the ASDEX Upgrade tokamak. It is found that fast reconnection observed during amplitude drops of the neoclassical tearing mode instability in the frequently interrupted regime can be related to stochastization. It is also shown that stochastization can explain the fast loss of confinement during the minor disruption. This demonstrates that stochastization can be regarded as a possible cause for different MHD events in ASDEX Upgrade.

\section{Introduction}

An important goal of fusion research with magnetically confined plasmas is to maximize the achievable plasma pressure. In a tokamak, neoclassical tearing modes (NTMs), i.e. magnetic islands with poloidal (m) and toroidal (n) mode numbers driven unstable by the loss of bootstrap current inside the island, are of major concern as they are considered to be the most severe limitation to the maximum achievable plasma pressure. Developing of such magnetic islands require resistive reconnection of the magnetic field lines. The resistive reconnection assumes resistive diffusion of the magnetic field through the plasma which is rather slow process. It was found in ASDEX Upgrade that some MHD processes involving these tearing modes have much faster time scales compared to the resistive diffusion time $\left(\tau_{R}=\mu_{0} l^{2} / \eta_{p l} \approx 10 \mathrm{~ms}\right.$, where $l \approx 1 \mathrm{~cm}$ is the characteristic length of the resistive layer). These processes involve interaction of several modes and can be explained by stochastization of the magnetic field lines. Stochastization means that for the trajectory of an object time averaging and spatial averaging can be exchanged. This means also that the trajectory of an object starting from any point in a stochastic area comes infinitely close to any other point of this volume.

In this paper we discuss in detail two such examples, namely the frequently interrupted regime of neoclassical tearing mode (FIR-NTM) and the minor disruption due to the interaction of the $(2,1)$ and $(3,1)$ tearing modes. During the FIR-NTM regime, the amplitude of the NTM periodically decreases to a much smaller value and never reaches its saturated value. The time in which these amplitude drops occur is very short (about $500 \mu s$ ), much shorter than the resistive MHD reconnection rate (few tens of milliseconds in the ASDEX Upgrade). In particular it has been observed that the amplitude of the $(m, n)=(3,2)$ NTM drops as an additional MHD instability (the $(\mathrm{m}, \mathrm{n})=(4,3)$ mode) occurs $[1,2]$. Generally the occurrence of the $(\mathrm{m}+1, \mathrm{n}+1)$ modes always coincides with the $(1,1)$ mode activity which is a necessary condition for the nonlinear coupling to the $(\mathrm{m}, \mathrm{n})$ NTM. Moreover, the sudden drops in NTM amplitude only occur if the three modes are locked in phase. The ideal $(4,3)$ mode needs to be driven unstable by non-linear mode 
coupling [3]. Increasing the plasma pressure gradient has a destabilizing effect on this mode and thus makes a non-linear destabilization easier. This might be the reason for the required high plasma pressures for FIR-NTMs [4,5]. The other example is the minor disruption in ASDEX Upgrade. Experiments show that interaction of $(2,1)$ and $(3,1)$ tearing modes leads to a sequence of minor disruptions [6]. This demonstrates that stochastization can be very important in cases of simultaneous activity of several MHD modes.

This work can be regarded as an extended and improved version of a recent letter [7]. In particular, new more realistic and physically justified parametrizations for perturbations of the $(1,1)$ and $(4,3)$ modes are introduced, interaction of $(3,1)$ and $(2,1)$ tearing modes is analyzed, mathematical details related to the mapping technique and to the characterization of degree of stochastization are presented. The work consists of five sections. In section 2, we recall some basic information about the Hamiltonian formalism and the mapping technique and present the specific mapping used in the present analysis. The experimental data and their parametrization are described in section 3. In section 4 we present the results of calculations based on the mapping introduced in section 2 and on the parametrizations adopted in section 3. Section 5 is devoted to quantitative aspects of stochasticity. Finally, in section 6 we summarize the main results and outline guidelines for the future work.

\section{Hamiltonian formalism and mapping technique}

Magnetic field lines can be regarded as trajectories of Hamiltonian systems. For the field line tracing two methods can be applied: i) integration of the trajectory and ii) mapping of the trajectory. The latter is a modern technique for the Hamiltonian system $[8,9,10]$ and is implemented in our code. It is more than an order of magnitude faster than the integration. A properly chosen mapping procedure always conserves the main flux preserving property of the magnetic field, which is important for a correct reproduction of the long-term behaviour of field lines in stochastic regions. In this formalism the equations for magnetic field lines take the Hamiltonian form

$\frac{d \psi}{d \varphi}=-\frac{\partial H}{\partial \vartheta}, \quad \frac{d \vartheta}{d \varphi}=\frac{\partial H}{\partial \psi}$,

where $\psi=\frac{r^{2}}{2 a^{2}}$ is a toroidal magnetic flux canonically conjugated to the poloidal angle $\vartheta, \varphi$ is a toroidal angle, and $a$ is a minor radius of the plasma (50 $\mathrm{cm}$ at ASDEX Upgrade). The Hamiltonian $H$

$H=H_{0}(\psi)+H_{1}(\psi, \vartheta, \varphi)$

can be represented as a sum of the unperturbed flux

$H_{0}(\psi)=\int \frac{d \psi}{q(\psi)}$

and the perturbed part of the flux 
$H_{1}(\psi, \vartheta, \varphi)=\sum_{m, n} H_{m n}(\psi) \cos \left(m \vartheta-n \varphi+\chi_{m n}\right)$

Here $q(\psi)$ is the safety factor characterizing the winding of the magnetic field lines, $H_{m n}(\psi)$ is the perturbation Hamiltonian which corresponds to the perturbations of the modes $(\mathrm{m}, \mathrm{n})$ with the phases $\chi_{m n}$.

For our purposes we have chosen the symmetric symplectic mapping derived in [9] on the basis of the Hamilton-Jacobi method. In the first order approximation this mapping can be written as follows:

$$
\begin{aligned}
& \Psi_{k}=\psi_{k}-\frac{\partial S^{(k)}}{\partial \vartheta_{k}} \\
& \Theta_{k}=\vartheta_{k}+\frac{\partial S^{(k)}}{\partial \Psi_{k}} \\
& \bar{\Theta}_{k}=\Theta_{k}+w\left(\Psi_{k}\right)\left(\varphi_{k+1}-\varphi_{k}\right) \\
& \Psi_{k+1}=\Psi_{k} \\
& \vartheta_{k+1}=\bar{\Theta}_{k}-\frac{\partial S^{(k+1)}}{\partial \Psi_{k+1}} \\
& \psi_{k+1}=\Psi_{k+1}+\frac{\partial S^{(k+1)}}{\partial \vartheta_{k+1}}
\end{aligned}
$$

where $w(\Psi)=1 / q(\Psi)$ is the spatial frequency of the perturbed motion and $S^{(k)} \equiv S\left(\vartheta_{k}, \Psi_{k}\right)$ is the value of the generating function $G\left(\vartheta, \Psi, \varphi, \varphi_{0} ; \varepsilon\right)$ taken at sections $\varphi=\varphi_{k}$, i.e. $S\left(\vartheta_{k}, \Psi_{k}\right)=G\left(\vartheta_{k}, \Psi_{k}, \varphi_{k}, \varphi_{0}\right)$. The first order generating function in the finite interval $\varphi_{k} \leq \varphi<\varphi_{k+1}$ is given by the expression $G\left(\vartheta, \Psi, \varphi, \varphi_{0}\right)=-\left(\varphi-\varphi_{0}\right) \sum_{m n} H_{m n}(\Psi)$ $\times\left[a\left(x_{m n}\right) \sin \left(m \vartheta-n \varphi+\chi_{m n}\right)+b\left(x_{m n}\right) \cos \left(m \vartheta-n \varphi+\chi_{m n}\right)\right]$

where $a(x)=[1-\cos (x)] / x, b(x)=\sin (x) / x, x_{m n}=(m / q(\Psi)-n)\left(\varphi-\varphi_{0}\right)$, and $\chi_{m n}$ are phases. For the symmetric mapping the free parameter $\varphi_{0}$ is taken exactly in the middle of the interval $\left[\varphi_{k}, \varphi_{k+1}\right]$, i.e. $\varphi_{0}=\left(\varphi_{k}+\varphi_{k+1}\right) / 2$.

\section{Parametrizations for perturbations}

It is obvious that practical implementation of the mapping method requires knowledge of the safety factor and of the perturbation Hamiltonian. Determination of these quantities from the experiment is a challenging task, because of the large uncertainties in the measurements.

We have chosen the following parametrization for the safety factor:

$$
q(\psi)=0.8+4 \psi
$$


which describes correctly the experimental position of the MHD modes in ASDEX Upgrade.

The perturbation amplitude $\varepsilon_{m n}$ in the Hamiltonian for each individual mode is defined as follows [11]:

$$
\varepsilon_{m n}=B_{m n} / B_{T}
$$

where $B_{T}$ is the primary toroidal magnetic field and $B_{m n}$ is the magnetic perturbation due to the $(\mathrm{m}, \mathrm{n})$ mode. It can be roughly estimated from the width of the magnetic islands on the basis of the standard formula [12]

$\Delta r_{m n}=4\left[\left.\frac{R_{0}}{m} \frac{B_{m n}(r)}{B_{T}}\left(\frac{d q^{-1}(r)}{d r}\right)^{-1}\right|_{r=r_{m n}}\right]^{1 / 2}$

and used in Eq. (13) for determination of $\varepsilon_{m n}$. In Eq. (14) $R_{0}$ is the major plasma radius. In normalized units $R_{0}=($ major radius $/$ minor radius $)=165 \mathrm{~cm} / 50 \mathrm{~cm}=3.3$. In Table I we summarize the average parameters of the three islands observed in the experiment.

Table I. Island width and corresponding perturbations.

--Double line under caption-

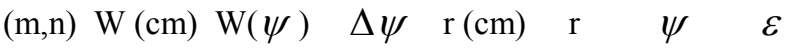

--Single line under headings-

$\begin{array}{llllllll}(1,1) & 5 & 0.10 & 0.0050 & 15.8 & 0.316 & 0.050 & 2.4 \times 10^{-4} \\ (3,2) & 8 & 0.16 & 0.0128 & 29.6 & 0.592 & 0.175 & 1.5 \times 10^{-3} \\ (4,3) & 3 & 0.06 & 0.0018 & 25.8 & 0.516 & 0.133 & 3.2 \times 10^{-4}\end{array}$

--Double line under the end of the table

Approximation (14) becomes rather inaccurate for small perturbations due to large error bars for the island width $\left(\Delta r_{m n}\right)$. Thus we try to estimate $B_{m n}(r)$ from magnetic measurements. As a first approximation, a representation of perturbation due to MHD mode can be written in the power law form [13,14]:

$$
\begin{aligned}
& H_{m n}=\varepsilon_{m n}\left(\frac{\psi}{\psi_{m n}}\right)^{m / 2} \text { for } \psi \leq \psi_{m n} \\
& H_{m n}=\varepsilon_{m n}\left(\frac{\psi}{\psi_{m n}}\right)^{-m / 2} \text { for } \psi>\psi_{m n}
\end{aligned}
$$

where $\psi_{m n}$ is the rational magnetic surface of the $(\mathrm{m}, \mathrm{n})$ mode. This representation corresponds to the socalled "step current" approximation (SCA), which assumes that the perturbation current due to the mode 
has a step function structure and is strongly localized at the corresponding resonant surface with $q=m / n$. Such an assumption can be used as the zero order approximation for any type of MHD activities with resonant surfaces inside the plasma. SCA gives the correct asymptotic behaviour $\left(\psi \sim r^{-m}\right)$, but it cannot describe the perturbed flux close to the resonant surface [15] (see Fig. 1).
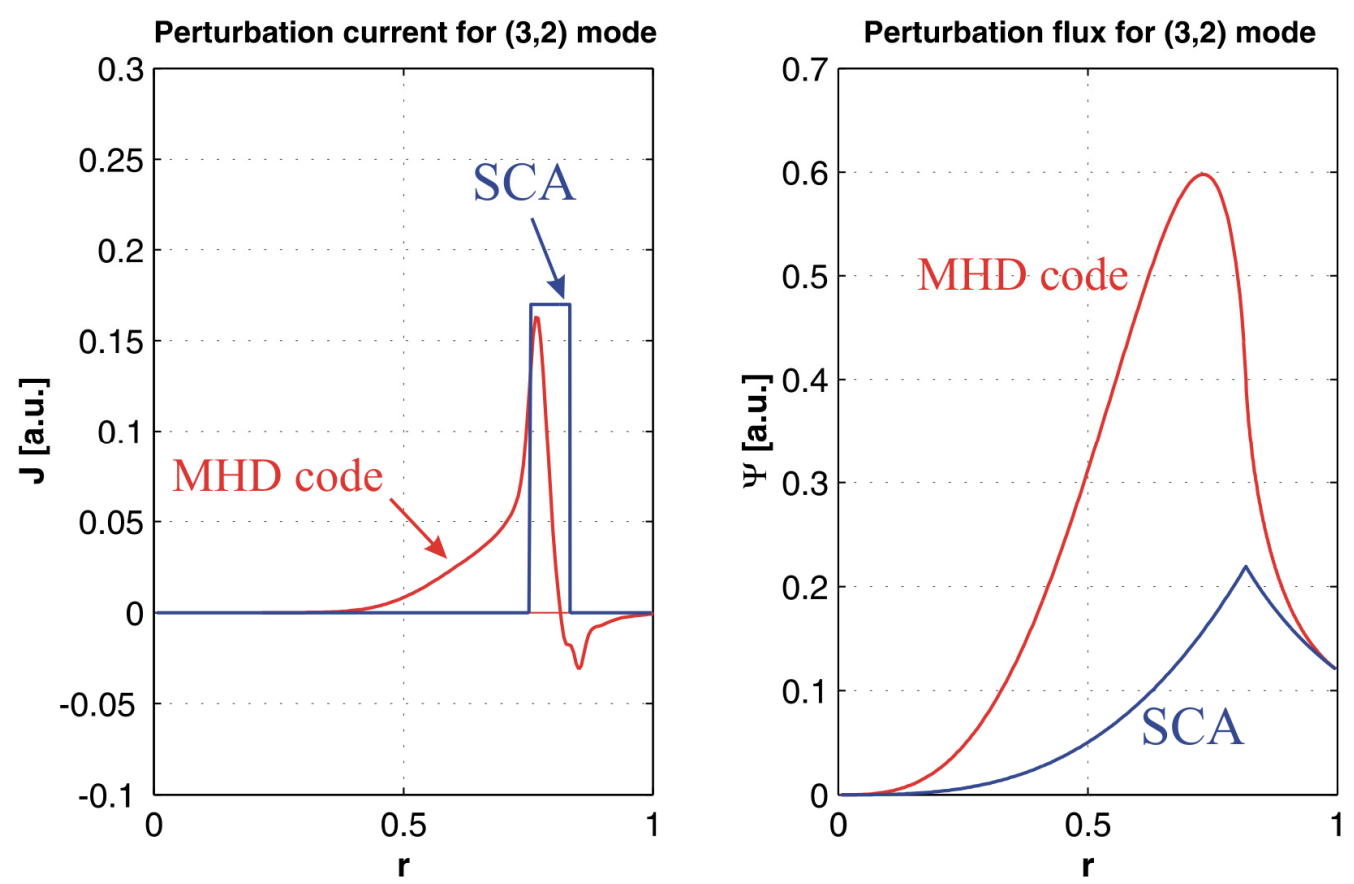

Figure 1. Perturbation currents and perturbation fluxes for the $(3,2)$ mode resulting from the tearing mode equation (red curves) and from the SCA approximation (blue curves). A significant difference between these two approaches $\left(r_{r e}\right.$ $=0.81)$ is evident. (This figure is from [15].)

The MHD simulations, as well as the electron cyclotron emission (ECE) measurements, show a completely different behaviour of the perturbation flux [16]. This difference can be attributed to the plasma influence which screens all the magnetic perturbations inside the plasma. This screening effect of the plasma is not taken into account in SCA which always underestimates the flux and hence has to be improved. Parametrization (15) has two drawbacks: i) incorrect shape of the perturbation flux close to the resonant surface, ii) large errors of $\varepsilon_{m n}$ in the case of small perturbations. These defects can be eliminated by using the experimental information from ASDEX Upgrade. We adopt the parametrization used in [16] for analyzing the structure of the $(3,2),(4,3)$ and $(5,4)$ modes 


$$
\begin{aligned}
& H_{m n}=\rho_{m n} \alpha\left(\frac{\psi}{\psi_{m n}}\right)^{m / 2}\left[1-\beta\left(\frac{\psi}{\psi_{m n}}\right)^{1 / 2}\right] \text { for } \psi \leq \psi_{m n} \\
& H_{m n}=\rho_{m n} \frac{\alpha(1-\beta)-\gamma+\gamma\left(\frac{\psi}{\psi_{m n}}\right)^{1 / 2}}{\left(\frac{\psi}{\psi_{m n}}\right)^{(m+1) / 2}} \text { for } \psi>\psi_{m n} \cdot
\end{aligned}
$$

Here $\alpha, \beta$ and $\gamma$ are free parameters which fix the shape of the perturbation flux. The values of these parameters have been determined in [16] from the analysis of ECE measurements of the $(3,2)$ mode and provide us a correct form of the perturbations which is a good approximation for resistive MHD modes. The perturbed flux for a resistive mode does not change sign at the resonant surface (see Fig.1). The situation becomes different for ideal modes. In this case, the perturbed flux changes the sign at the resonant surface. At the same time, the ideal MHD force balance equation is valid for both the ideal case and the resistive case (except in a small resistive layer). Thus, one would expect similar behaviour of the flux function for the two cases. For an ideal MHD mode we introduce a parametrization similar to (16) but with a sign jump at the resonant surface:

$$
\begin{aligned}
& H_{m n}=\rho_{m n} \alpha\left(\frac{\psi}{\psi_{m n}}\right)^{m / 2}\left[1-\beta\left(\frac{\psi}{\psi_{m n}}\right)^{1 / 2}\right] \cdot \frac{2 \cdot \operatorname{arctg}\left(\left(\psi-\psi_{m n}\right) \cdot \mu\right)}{\pi} \quad \text { for } \psi \leq \psi_{m n} \\
& H_{m n}=\rho_{m n} \frac{\alpha(1-\beta)-\gamma+\gamma\left(\frac{\psi}{\psi_{m n}}\right)^{1 / 2}}{\left(\frac{\psi}{\psi_{m n}}\right)^{(m+1) / 2}} \cdot \frac{2 \cdot \operatorname{arctg}\left(\left(\psi-\psi_{m n}\right) \cdot \mu\right)}{\pi} \text { for } \psi>\psi_{m n},
\end{aligned}
$$

Here the additional parameter $\mu$ determines the width of the resonant region. Typically $\mu$ is about 500 which corresponds to the resonant layer about 3 percent of the plasma radius.

The perturbation amplitudes $B_{m n}$ can be directly deduced from the magnetic measurements which give us magnetic perturbations at the position of the magnetic probes located outside the plasma at $r=1.3 \cdot a$, where $a$ is the minor plasma radius $(\psi=0.845)$. The normalization coefficients $\rho_{m n}$ can be determined by demanding that the values of $H_{m n}$ given by Eqs. (16) and (17) coincide with the measured values at the position of the probes. In other words:

$$
H_{m n}(\psi=0.845)=H_{m n}^{\exp }(\psi=0.845)
$$

In tokamaks, instabilities at the $q=1$ rational surface have a completely different structure compared to other internal resonant surfaces [17]. The $(1,1)$ mode should have an ideal character in order to 
be coupled to the resistive NTM. Thus, a new special parametrization for the ideal $(1,1)$ mode has to be derived. It is a common knowledge that any MHD mode can be characterized by the incompressible helical displacement $\xi$ of the equilibrium magnetic flux surfaces. The shape of this displacement for the $(1,1)$ kink mode is well known:

$$
\xi(r)= \begin{cases}\xi_{0}, & r<r_{(11)} \\ 0, & r \geq r_{(11)}\end{cases}
$$

where $\xi_{0}$ is the amplitude of the displacement inside the $q=1$ resonant surface. The relation between the displacement and the perturbed flux follows from the Ohm's law:

$\Psi_{(1,1)}(r)=\xi(r) \frac{r B_{T}}{R_{0} q(r)}(1-q(r))$.

Thus, the perturbed part of the Hamiltonian has the form:

$$
H_{11}= \begin{cases}\xi_{0} \frac{\sqrt{2 \psi} B_{T}}{R_{0} q(\psi)}(1-q(\psi)), & \psi<\psi_{11} \\ 0, & \psi \geq \psi_{11}\end{cases}
$$

This mode is located at the plasma core where a reconstruction of the perturbation flux from ECE measurements is difficult. It also has a burst character during the FIR which leads to additional obstacles for reconstruction of the mode structure. At the same time, ECE measurements for the $(1,1)$ fishbones in ASDEX Upgrade gives us values for the displacement about $1-3 \mathrm{~cm}$. Thus, we can use $\xi_{0}=3 \mathrm{~cm}$ as an upper limit for the $(1,1)$ perturbation amplitude.

The resulting perturbations are shown in Fig. 2. It should be noted that the parameterization for the resistive $(1,1)$ mode would have almost the same shape. The only difference would come from the small negative part for the displacement close to $\mathrm{q}=1$ resonant surface. Perturbations which are use in the next section are summarized in Table II.

Table II. MHD modes, their characteristics and parameterizations.

--Double line under caption-

$(\mathrm{m}, \mathrm{n})$ Mode character Parameterization equation Type of MHD activity

--Single line under headings-

$\begin{array}{lccc}(1,1) & \text { ideal } & 21 & \text { all FIR-NTMs cases } \\ (3,2) & \text { resistive } & 16 & \text { FIR-NTM }(1,1)+(3,2)+(4,3) \\ (4,3) & \text { resistive } & 16 & \text { FIR-NTM }(1,1)+(4,3)+(5,4) \\ (4,3) & \text { ideal } & 17 & \text { FIR-NTM }(1,1)+(3,2)+(4,3) \\ (5,4) & \text { ideal } & 17 & \text { FIR-NTM }(1,1)+(4,3)+(5,4) \\ (2,1) & \text { resistive } & 16 & \text { Disruption }(2,1)+(3,1)\end{array}$


--Double line under the end of the table

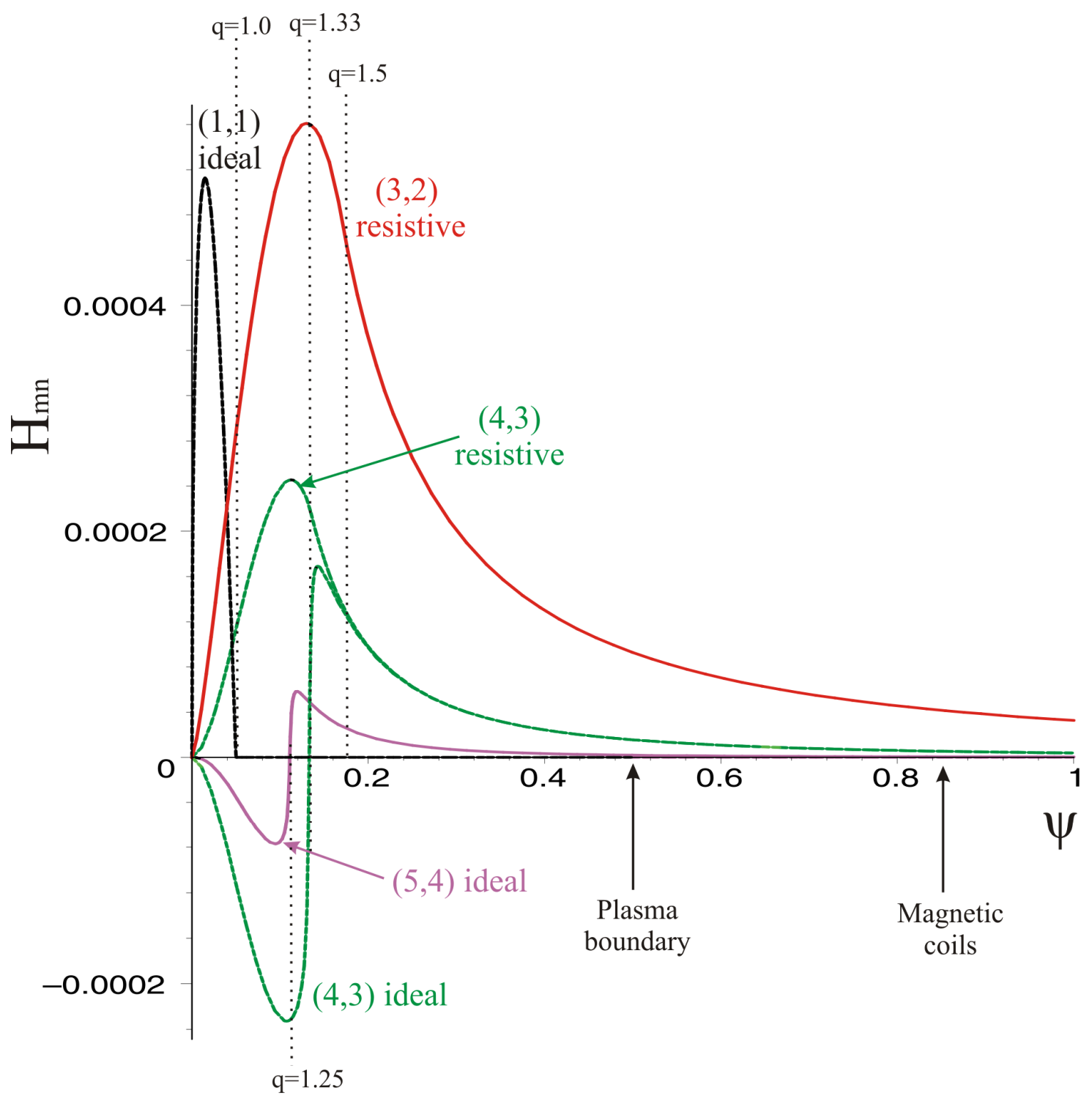

Figure 2. Experimental perturbations for the $(1,1),(3,2),(4,3)$ and $(5,4)$ modes as a function of magnetic flux for the ASDEX

Upgrade discharge \#11681, $\mathrm{t}=2.98 \mathrm{~s}$. The parametrization for the $(4,3)$ mode is shown for both the ideal and the resistive variants of the instability. The parametrization for $(5,4)$ mode is shown only for the ideal instability. Here $\alpha=0.04, \beta=0.87, \gamma=0.005$, and $\mu=500$ for the $(3,2),(4,3)$ and $(5,4)$ modes, $H_{32}^{\text {exp }}=4.2 \times 10^{-5}, H_{43}^{\exp }=5.4 \times 10^{-6}$, and $H_{54}^{\text {exp }}=5.0 \times 10^{-7}$. For the $(1,1)$ mode it is assumed that the displacement $\xi_{0}=3 \mathrm{~cm}$. The plasma boundary and the position of the magnetic coils are indicated by the arrows. The dotted vertical lines mark the positions of resonance surfaces. 
It is interesting to compare the value $H_{32}^{\text {exp, max }}=4.5 \times 10^{-4}$, given by parametrization (15) and the values $H_{32}^{\text {exp, max }}=5.6 \times 10^{-4}$ given by parametrization (16) with the amplitude of the perturbations obtained from the island widths (Table I). SCA predicts the width of the island corresponding to the $(3,2)$ mode of the order of $4.2 \mathrm{~cm}$, while parametrization (16) gives about $5.1 \mathrm{~cm}$ which is closer to the value $5.2 \mathrm{~cm}$ obtained in an independent ECE measurement [2]. This fact confirms that our parametrization describes the perturbed flux better then the simple SCA assumption.

\section{Analysis of the ASDEX Upgrade data}

In this section, the mapping technique is applied to investigate the stochastization between different modes for several experimental cases. Perturbations for the Hamiltonian formalism are expressed in terms of the experimental perturbation amplitudes determined from the ASDEX Upgrade tokamak data. We consider here the FIR of NTM and the minor disruption due to the interaction of the $(3,1)$ and $(2,1)$ modes. We show that stochastization can describe these two different cases.

\section{Frequently interrupted regime of the neoclassical tearing modes}

The results of the calculations performed with parametrization (21) for the $(1,1)$ mode, parametrization (16) for the $(3,2)$ mode, and parametrization $(17)$ for the $(4,3)$ mode are shown in Figs. 3,4 and 5.

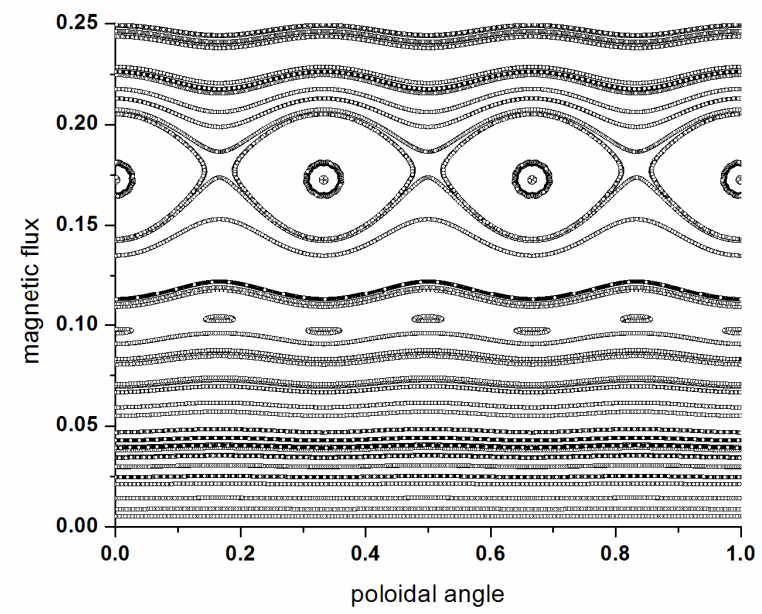

Figure 3. The $(3,2)$ resistive mode is used as a perturbation. Shape of the perturbation is shown in Fig. 2. The ASDEX Upgrade discharge \#11681, $\mathrm{t}=2.98 \mathrm{~s}$ 


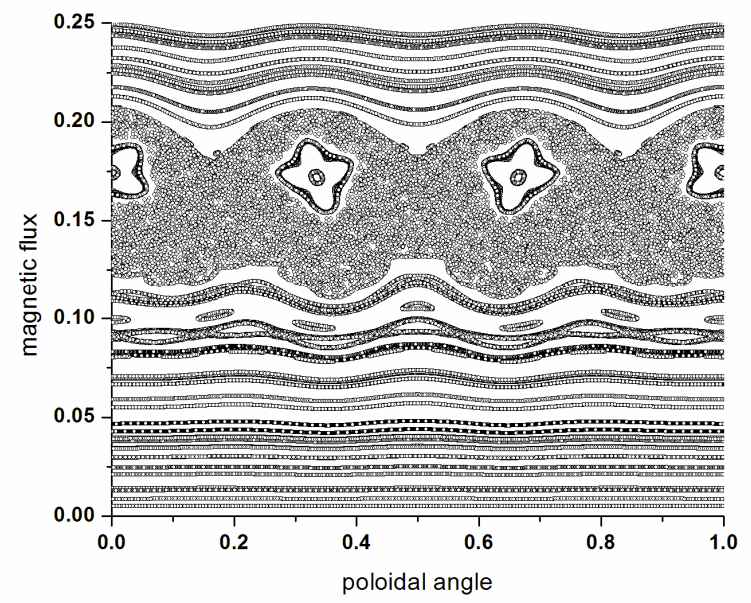

Figure 4. The $(3,2)$ resistive and $(4,3)$ ideal modes are used as perturbations. Shapes of the perturbations are shown in Fig. 2. The ASDEX Upgrade discharge No.11681, $\mathrm{t}=2.98 \mathrm{~s}$.

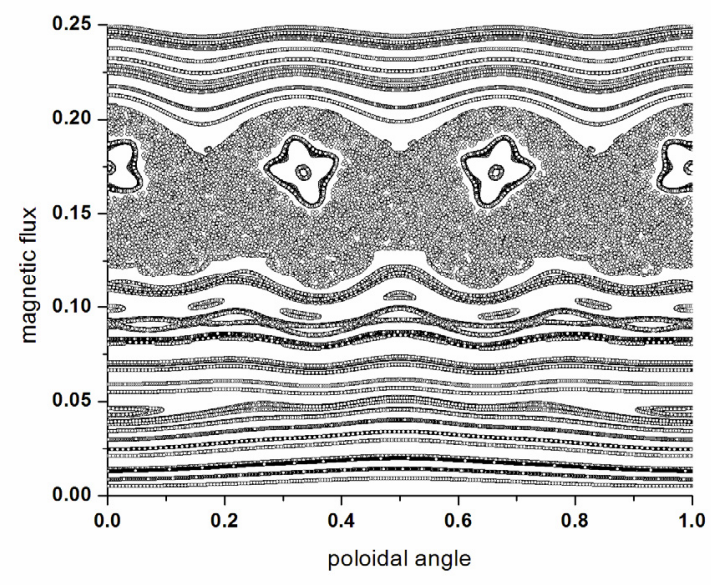

Figure 5. The $(1,1)$ ideal, $(3,2)$ resistive and $(4,3)$ ideal modes are used as perturbations. Shapes of the perturbations are shown in Fig. 2. The ASDEX Upgrade discharge No.11681, $\mathrm{t}=2.98 \mathrm{~s}$.

The Poincare sections of the field lines shown in Figs. 4 and 5 correspond to the time point when the modes are locked together. The presence of the $(4,3)$ mode immediately produces a large region of stochastisity (Figs. 4 and 5). This is due to the fact that perturbations of individual modes strongly overlap (Fig. 2). This overlapping is especially pronounced in the case of the $(3,2)$ and $(4,3)$ modes, because the distance between the resonant surfaces of these two modes is very small. On the one hand, the influence of the $(1,1)$ mode on the stochastization itself is very weak (compare Fig. 4 with Fig. 5), because $H_{11}(\psi)=0$ for $\psi>\psi_{11}$. Here variations of the amplitude and the shape of this mode within the experimental errors do not change the overall picture. On the other hand, the presence of the $(1,1)$ mode is needed for providing a 
nonlinear coupling between all the modes at the time point, when the modes are mutually locked and the stochastic region develops. As pointed out in Ref. 1, even a large (4,3) amplitude just before the occurrence of the mode coupling does not reduce the $(3,2)$ amplitude. It drops only after the phase velocities of the $(3,2)$ and $(4,3)$ modes become equal. We conclude that our calculations support the anticipation that the nonlinear interaction between the $(3,2)$ and $(4,3)$ modes leads to stochastization and that the presence of the $(4,3)$ mode really is able to prevent the $(3,2)$ mode from growing to its saturated island size.

It was found in ASDEX Upgrade that the FIR is not restricted to the interaction between the $(3,2)$ and $(4,3)$ modes. The same phenomenon has been observed in the case of the resistive NTM $(4,3)$ and the ideal $(5,4)$ modes. The results of the calculations for these modes are shown in Fig. 6.

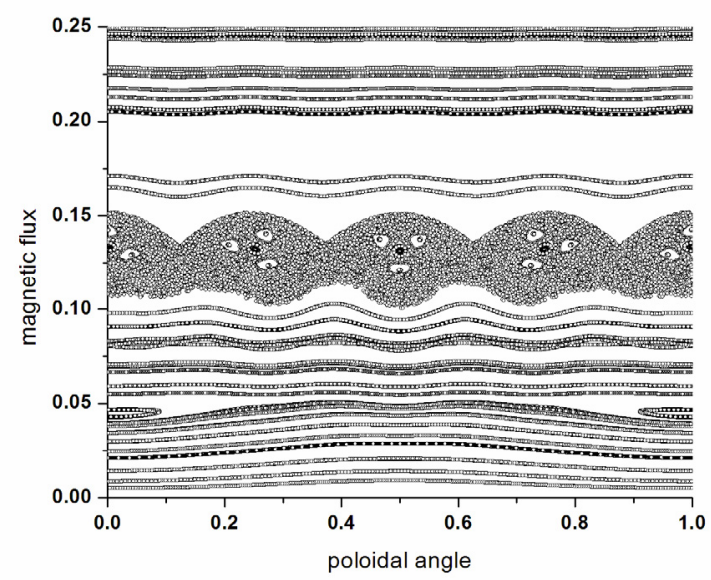

Figure 6. The $(1,1)$ ideal, $(4,3)$ resistive, and $(5,4)$ ideal modes are used as perturbations. The ASDEX Upgrade discharge \#11681, $\mathrm{t}=2.66 \mathrm{~s}$.

The physics of the interaction is exactly the same as in the case of the $(3,2)$ and $(4,3)$ modes. The only difference is the critical value of the amplitude for the $(5,4)$ mode which is needed for creation of a stochastic region between the $(4,3)$ and $(5,4)$ modes. It was found that this critical value is an order of magnitude smaller than the experimental value! The reason for this is a very small distance between the $(4,3)$ and $(5,4)$ modes compared to the distance between the $(3,2)$ and $(4,3)$ modes. The resonances in this case completely overlap and even a tiny perturbation leads to stochastization. The stochastization does not occur, if the amplitude of the $(5,4)$ mode is 20 times smaller than the experimental value. As was already pointed out in the previous example, to create a stochastic region one has to fulfil two conditions. First, the amplitude of the $(5,4)$ mode must be larger than the threshold value, which indeed is the case. Second, all the modes have to be locked simultaneously. Unless both of these conditions are fulfilled, stochastization does not occur. Thus, for the $(4,3)$ NTM, clearly the locking condition determines the onset.

Our calculations also show that interaction of $(3,2)$ NTM with ideal $(4,3)$ mode gives practically the same picture as an interaction of $(3,2)$ NTM with resistive $(4,3)$ mode (for the same set of the parameters: $\alpha, \beta, \gamma, \mathrm{H}_{m n}^{\exp }$ as shown in Fig.2 for $(4,3)$ mode). This is an expected result because perturbations with 
different helicities can not cancel each other even if these perturbations have different signs (see Fig.2 for $(3,2)$ resistive and $(4,3)$ ideal modes inside the $(4,3)$ resonant surface). The stochastization depends mainly on the amplitudes of these overlapping harmonics. Thus, it is not important for stochastization whether the $(4,3)$ mode has a resistive or an ideal character. The same conclusion is valid for the interaction of the $(4,3)$ NTM and the $(5,4)$ mode.

\section{Minor disruption due to the interaction of the $(3,1)$ and $(2,1)$ tearing modes}

It was observed in ASDEX Upgrade discharge that series of minor disruptions are accompanied by the interaction of the $(3,1)$ and $(2,1)$ modes [6]. Such a minor disruption leads to temporary deterioration of confinement and flattening of the temperature profile. We have modeled this disruption by using the perturbation amplitude obtained by means of ECE measurements, by assuming that parametrization (16) is valid for these two tearing modes and by adjusting the safety factor to the measured positions of the rational surfaces (Fig. 7).

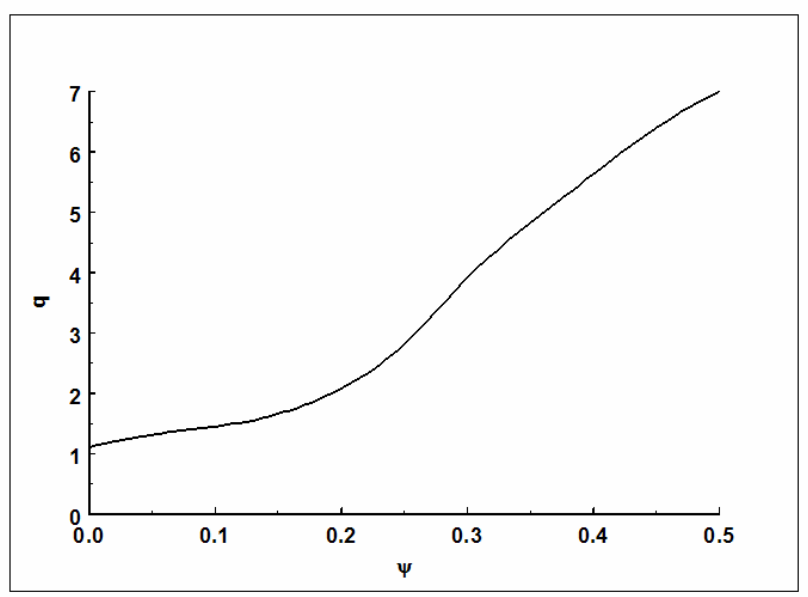

Figure 7. Safety factor as a function of the magnetic flux as defined by Eq. (1).

The results of the calculations are shown in Figs. 8-11. 


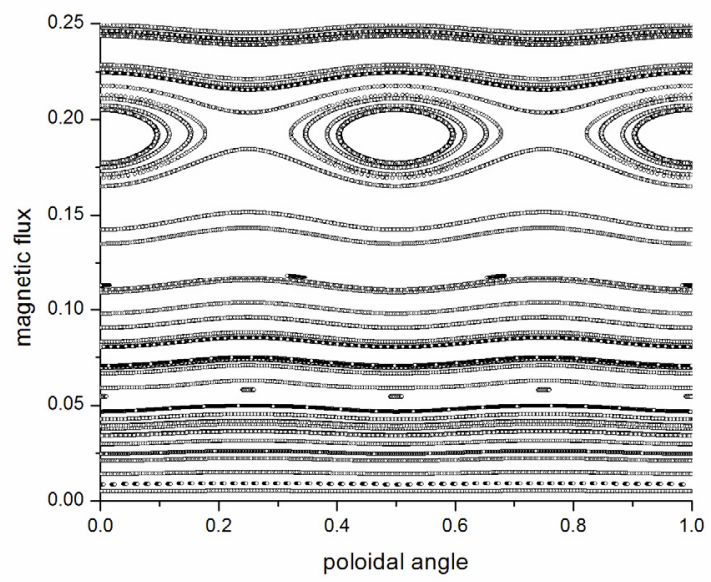

Figure 8. The $(2,1)$ resistive mode is used as a perturbation. The amplitude of the perturbation 0.00008 is chosen such that the island width is equal to the experimental value $0.05 \mathrm{~m}$, as seen in Fig. 2 of Ref. 16 . Here $r[m]=0.707 \sqrt{\psi}$. The ASDEX Upgrade discharge \#6353, $\mathrm{t}=1.74 \mathrm{~s}$

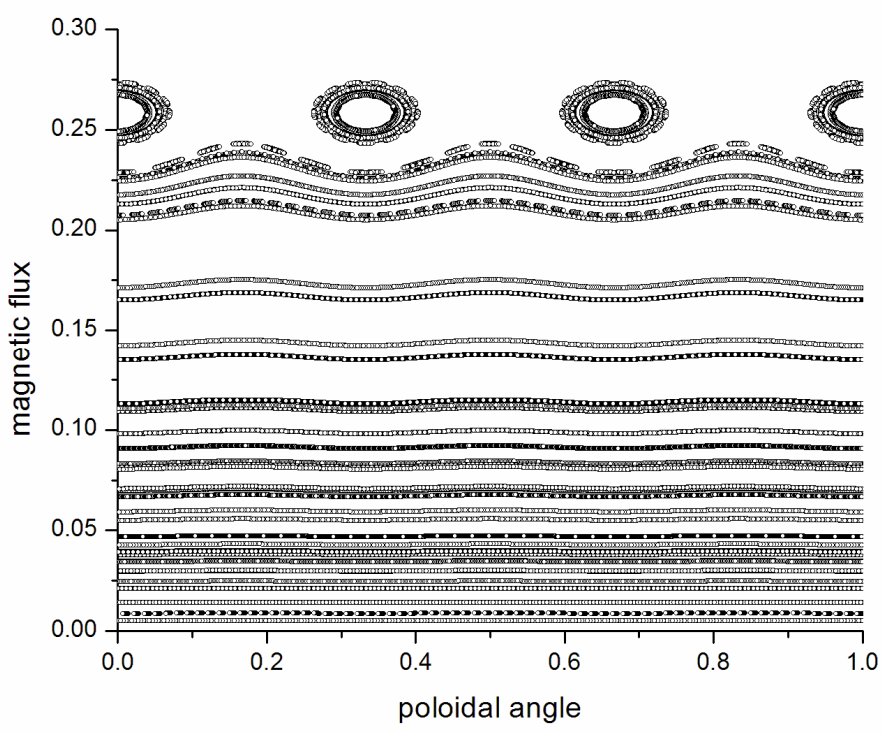

Figure 9. The $(3,1)$ resistive modes is used as a perturbation. The amplitude of the perturbation 0.00006 is chosen such that the island width is equal to the experimental value $0.02 \mathrm{~m}$, as seen in Fig. 2 of Ref. 16. Here $r[m]=0.707 \sqrt{\psi}$.The ASDEX Upgrade discharge \#6353, $\mathrm{t}=1.74 \mathrm{~s}$. 


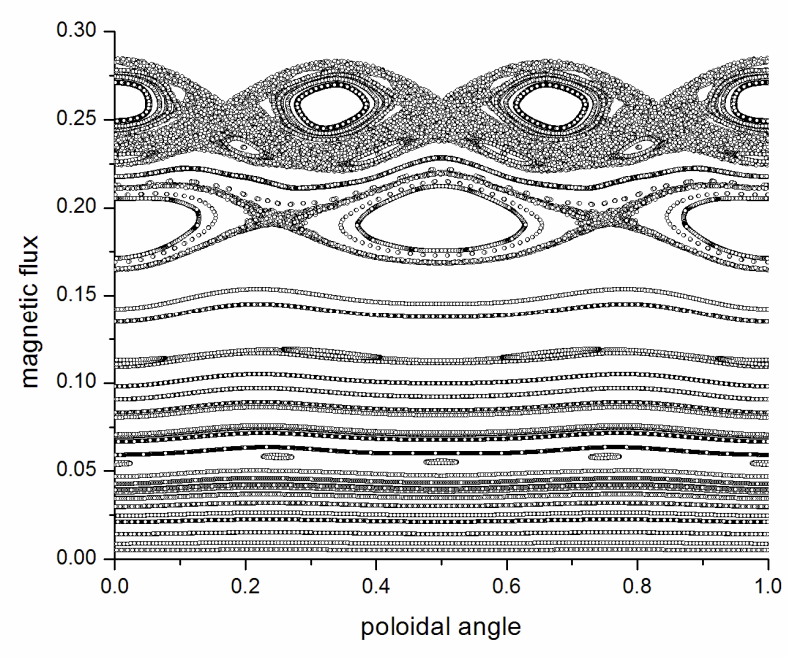

Figure 10. The $(2,1)$ and $(3,1)$ modes are used as perturbations. Amplitudes of the modes are the same as in Figs. 8 and 9. The ASDEX Upgrade discharge \#6353, $\mathrm{t}=1.74 \mathrm{~s}$.

These modes are always coupled and one can observe stochastization around the islands (Fig. 10). However, if the amplitude of the $(2,1)$ is slightly increased $(0.00008 \rightarrow 0.00010)$, also the region between the modes is stochastized (Fig. 11) which means that in this case we have a trigger problem.

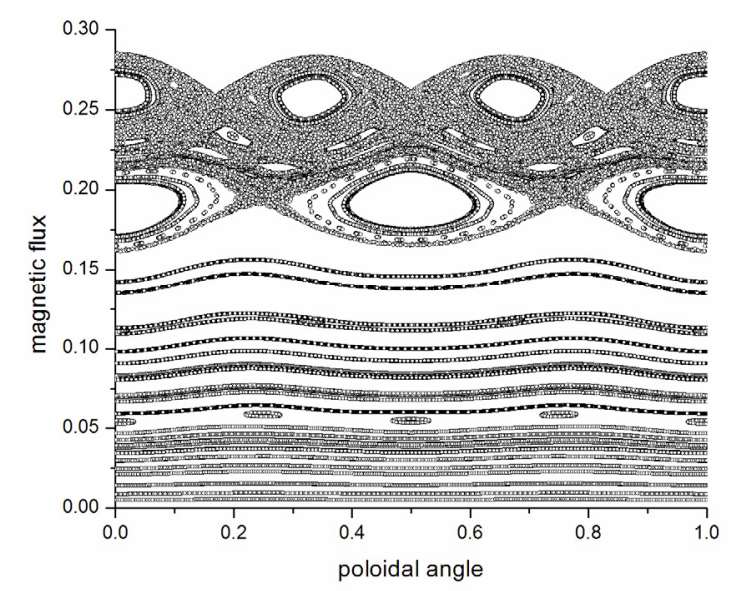

Figure is seen 11. Same as Fig. 11, but with a larger $(2,1)$ amplitude.

One would expect that such a stochastization destroys the confinement between the corresponding resonant surfaces and flattens the temperature profile which is observed in the ECE measurements. This case is completely different as compared to the FIR-NTM case. For the FIR-NTM the perturbation amplitudes are always large enough to create a stochastic region, but stochastization takes place only during the coupling period between the two modes. 


\section{Quantitative aspects of stochastisity}

In this section we address some general questions related to stochastization using the example of interaction of the $(3,2)$ and $(4,3)$ modes: taken the magnitude of the $(3,2)$ perturbation amplitude from the experiment, is there a lower limit of the $(4,3)$ perturbation amplitude for the onset of stochastization? Why and how does the stochastic zone increase when the $(4,3)$ perturbation amplitude increases? What are the special properties of the experimental $(4,3)$ perturbation amplitude? We will try to answer these questions using arguments from the theory of dynamical systems.

For a systematic study we keep the $(3,2)$ perturbation amplitude fixed to its experimental value and increase the $(4,3)$ perturbation amplitude in small steps starting from its zero value.

Two different approaches are used to generate a stochastic zone. First, it can be obtained as a union of many short stochastic trajectories starting from points inside the zone. This is used in the case of the Lyapunov exponent method. Second, it can be obtained by generating a single very long trajectory that wanders in the entire zone. This is used in the case of the coarse-grained method. The advantage of the first approach is that it provides a global understanding of the dynamics of the system in the short time evolution (5000 iterations). The second approach provides information of the complex evolution of a single magnetic line, hence it can be considered as a local dynamical description.

Let us first consider the system in the absence of the $H_{43}$ perturbation. It turns out that even in this case a thin chaotic layer surrounds the $(3,2)$ mode. This is illustrated in Fig. 12 where the first 100000 iterations are plotted of the trajectory starting from the initial point $(\pi / 3,0.175)$ which is located close to the hyperbolic periodic point of type $(3,2)$.

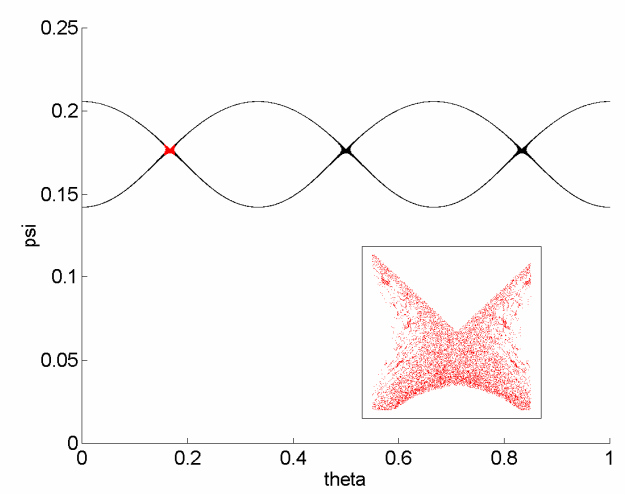

Figure 12. Phase portrait corresponding to the case when only the $(3,2)$ mode is used as a perturbation. The insert shows details of the small red region surrounding the hyperbolic point.

This observation is in agreement with the theory of area-preserving maps $[18,19]$. The perturbation $H_{32}$ is large enough to determine the transverse intersection of the stable and unstable manifolds of the hyperbolic 
periodic point, hence the formation of the stochastic layer. Even a small $(4,3)$ perturbation influences considerably the stochastization of the system. In Fig. 13 this is illustrated by means of the trajectory starting from $(\pi / 3,0.175)$.

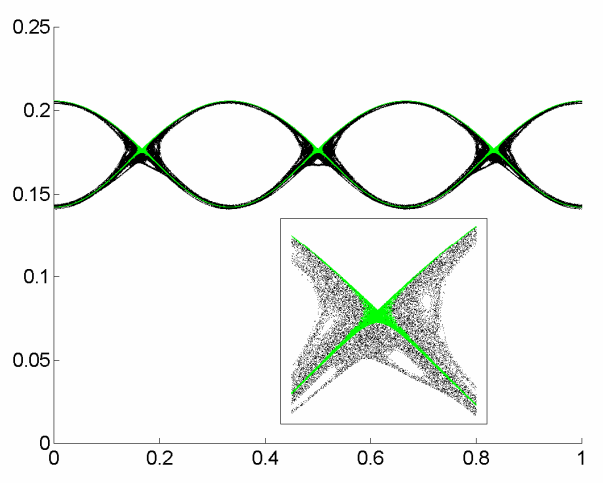

Figure 13. Phase portrait corresponding to the cases $H_{43}^{\text {exp }}=0$ (green points) and $H_{43}^{\text {exp }}=2 \cdot 10^{-7}$ (black points). The region surrounding the hyperbolic point is zoomed in the square.

With increasing the $(4,3)$ perturbation amplitude, the area of the $(3,2)$ mode decreases and the area of the stochastic zone increases. Stochasticity for a particular set of the $(3,2)$ and $(4,3)$ mode perturbations can also be studied on the basis of Lyapunov exponents. Here $\mathrm{N}_{\mathrm{T}}$ trajectories originating from random initial conditions $0 \leq \psi_{0}<0.25$ and $0 \leq \vartheta_{0} / 2 \pi<1$ are studied. The positive Lyapunov exponent $\lambda$ for each trajectory is calculated using the method described in [20], which is applicable to discrete maps and to Eqs. (5)-(10), in particular. One can rewrite the system of Eqs. (5)-(10) in the form $\vec{F}\left(\psi_{n+1}, \vartheta_{n+1}, \Psi_{n+1}\left(\psi_{n+1}, \vartheta_{n+1}\right), \psi_{n}, \vartheta_{n}, \Psi_{n}\left(\psi_{n}, \vartheta_{n}\right)\right)=0$, where $\vec{F}$ is a differentiable vector function. Then the Jacobian matrix can be obtained as

$J(n)=\left(\begin{array}{ll}\partial \psi_{n+1} / \partial \psi_{n} & \partial \psi_{n+1} / \partial \vartheta_{n} \\ \partial \vartheta_{n+1} / \partial \psi_{n} & \partial \vartheta_{n+1} / \partial \vartheta_{n}\end{array}\right)$

In general we can write $A_{n+1}=J(n) A_{n}$ where $A_{n}=J(n-1) \ldots J(1) J(0)$. The matrix $A_{n}$ can be decomposed into a product of an orthogonal matrix $\vec{Q}$ and an upper triangular matrix $\vec{R}$ with positive diagonal elements $\vec{Q}_{n+1} \vec{R}_{n+1}=J(n) \vec{Q}_{n} \vec{R}_{n}$. The Lyapunov exponents can be found from the diagonal elements of $\vec{R}_{n}$ 
$\lambda_{i i}=\lim _{n \rightarrow \infty} \frac{\ln \left(R_{n}\right)_{i i}}{n}$

where $i i=1,2$. Depending on the value of the maximal Lyapunov exponent the trajectory is marked as chaotic if $\lambda>\lambda_{\text {crit }}$, where $\lambda_{\text {crit }}$ is the critical value separating chaotic and non-chaotic trajectories. The stochastic area is defined as

$S=\lim _{N_{T} \rightarrow \infty}\left(N_{C} / N_{T}\right)$

where $N_{C}$ is the number of chaotic trajectories. The area $S$ can be interpreted as the probability that a randomly started trajectory becomes chaotic. First, we analyse the sensitivity of $S$ with respect to variation of the parameters $\lambda_{\text {crit }}, N_{T}$ and the number of iterations $N_{I}$ in the case of $H_{32}^{\exp }=4.2 \times 10^{-5}$ and $H_{43}^{\text {exp }}=5.4 \times 10^{-6}$. Here we choose $\lambda_{\text {crit }}=0.05$. For $\lambda<\lambda_{\text {crit }}$ the trajectory follows a closed path and is regarded as non-chaotic, otherwise it is regarded as chaotic. This is illustrated in Fig. 14.

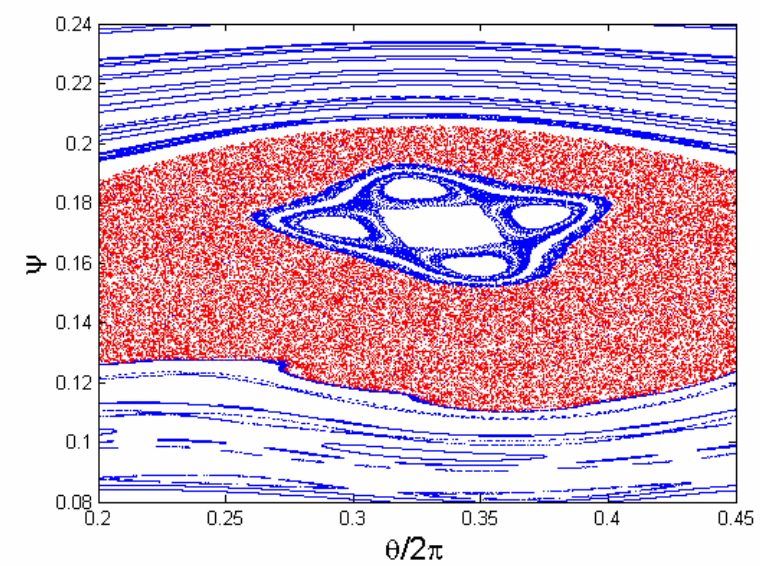

Figure 14. Snapshot of the phase space with chaotic (red) and non-chaotic (blue) trajectories separated by the definition $\lambda_{\text {crit }}=0.05$. Here the $(3,2)$ and $(4,3)$ modes are used as perturbations.

The robustness of $S$ with respect to variation of the parameter $\lambda_{\text {crit }}$ is illustrated by the observation that a variation of $\lambda_{\text {crit }}$ by $50 \%$ results in the change of $S$ by only $4 \%$ (Table III). Here the number of trajectories $N_{T}=10000$ and the number of iterations $N_{I}=5000$ are found to be optimal leading to saturated values of $S$.

Table III. Dependence of the stochastization area on parameters

--Double line under caption-

$\begin{array}{llll}N_{T} & N_{I} & \lambda_{\text {crit }} & S\end{array}$ 
--Single line under headings-

$\begin{array}{llll}10000 & 5000 & 0.050 & 0.256 \\ 10000 & 5000 & 0.025 & 0.266 \\ 10000 & 5000 & 0.075 & 0.250 \\ 1000 & 5000 & 0.050 & 0.263 \\ 20000 & 5000 & 0.050 & 0.253 \\ 10000 & 10000 & 0.050 & 0.258\end{array}$

--Double line under the end of the table

Next, we analyse the influence of the $(4,3)$ perturbation on stochastization for the fixed value of the $(3,2)$ perturbation amplitude. The results are shown in Fig 15 (black line). It is seen that the stochastic area grows almost linearly with the perturbation up to $H_{43}^{\exp }=1.1 \times 10^{-6}$. The linear growth with the subsequent saturation is observed for $(4,3)$ perturbation values $1.1 \times 10^{-6}-3.2 \times 10^{-6}$. Similar step-like behaviour is observed for $H_{43}^{\text {exp }}$ values in the range $3.2 \times 10^{-6}-4.9 \times 10^{-6}$. Here for the maximal $H_{43}^{\exp }$ value the probability that a randomly started trajectory becomes chaotic is $25 \%$.

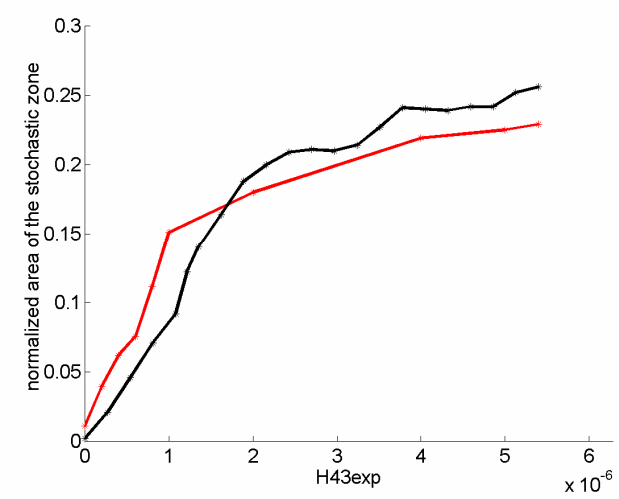

Figure 15. Dependence of the stochastic area on the magnitude of the $(4,3)$ perturbation amplitude. The black line corresponds to the Lyapunov exponent method and the red line represents the coarse-grained method.

It is remarkable that the values of $S$ obtained by means of the Lyapunov exponent method are in agreement with the values of the area computed using the coarse-grained method. In order to compare the results, the normalized coarse-grained areas, i.e. the current area divided by the area of $[0,1] \times[0,0.25]$, are drawn in figure 15 (red line) for various values of $H_{43}^{\text {exp }}$. The stochastic area was approximated by means of a grid of squares of the size 0.001 and 100000 points on the trajectory starting from $(\pi / 3,0.175)$ for all the perturbations. If $N$ is the number of squares needed to cover the chaotic trajectory, then the 
coarse-grained area is $N \cdot 10^{-6}$. Hence the normalized coarse-grained area is $N / 250000$. The area of the stochastic zone is overestimated in the coarse-grained method. For a region with the boundary of length $L$ the error of the estimate is of the order $L \cdot 0.001$. This fact explains why for small perturbations the red line is located above the black one. For large perturbations a single stochastic trajectory can cross with difficulty the entire stochastic zone. However, this zone can be covered by many short stochastic trajectories starting from points located in different places. In this situation the coarse-grained area becomes smaller than $S$. For this reason the red line is located below the black one in Fig. 15 for large values of $H_{43}^{\text {exp }}$. For longer trajectories the two approximations yield almost coinciding results. It is remarkable that the two alternative methods show that the system with increasing $H_{43}$ becomes more complex, more "chaotic, stochastic." The coarse-grained method being more direct but less sophisticated than the Lyapunov method emphasizes a different aspect of stochasticity.

A general theory $[18,19]$ can be used to explain the enlargement of the stochastic zone. Namely, with increasing perturbation some barriers separating the neighbouring modes are destroyed and several other modes are embedded into the same stochastic zone, i.e. into a region with positive area covered by trajectories starting from the points of this area. At the same time the sizes of the modes decrease, because some invariant curves situated inside them are also destroyed. The increase of the stochastic zone is larger when the newly incorporated $(m, n)$ mode has small period $m$. In order to identify the main modes contributing to the enlargement of the stochastic zone, we first consider the unperturbed system.

In the unperturbed system, corresponding to $H_{43}^{\exp }=H_{32}^{\exp }=0$, the periodic points that generate the $(m, n)$ mode are located on the circle $\psi=\psi_{m, n}$, where $\psi_{m, n}$ is the unique solution of the equation $q(\psi)=m / n$. Practically all the points of the circle $\psi=\psi_{m, n}$ rotate with $\theta_{m n}=2 \pi / q\left(\psi_{m n}\right)=2 \pi n / m$

Since the safety factor $q$ is an increasing function of $\psi$, the periodic points of the type $\left(m_{1}, n_{1}\right)$ are closer to the polar axis $\psi=0$ than the periodic points of the type $\left(m_{2}, n_{2}\right)$ if $m_{1} / n_{1}<m_{2} / n_{2}$. In between the periodic points of type $(m, n)$ with $m_{1} / n_{1}<m / n<m_{2} / n_{2}$ can be found.

In order to select the most important modes located between the two fixed points $\left(m_{1}, n_{1}\right)$ and $\left(m_{2}, n_{2}\right)$, one can use the Farey tree method [21]. This method is an interpolating scheme which 
organizes rational numbers $m / n$ into certain levels. Namely the $\left(m_{1}+m_{2}, n_{1}+n_{2}\right)$ mode located between the $\left(m_{1}, n_{1}\right)$ and $\left(m_{2}, n_{2}\right)$ modes has the smallest period. By means of this technique the classification shown in Table IV can be obtained.

Table IV. Locations of the principal $(m, n)$ modes

--Double line under caption-
$(\mathrm{m}, \mathrm{n})$
$(1,1)$
$(6,5)$

$(5,4)$

$(11,8)$

--Single line under headings-

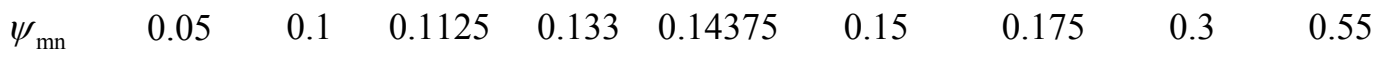

--Double line under the end of the table

With increasing $(4,3)$ perturbation amplitude one expects to observe incorporation of those principal modes into the stochastic zone which are located below the $(3,2)$ mode which remains dominant, because its amplitude is much larger than the amplitudes of other modes.

These observations are in agreement with the Chirikov resonance overlap criterion [22].

Indeed, the unperturbed island widths are $\delta_{32}=\left(0.8+4 \psi_{32}\right) \sqrt{\rho_{32} \cdot H_{32, \max }}$

and $\delta_{43}=\left(0.8+4 \psi_{43}\right) \sqrt{\rho_{43} \cdot H_{43, \max }}$. Here $H_{32, \text { max }} \approx 0.0064066$ corresponds to $\psi_{\max 32} \approx 0.1301$ and $H_{43, \max }=\rho_{43} \cdot 0.0039275$ corresponds to $\psi_{\max 43} \approx 0.143$ The Chirikov parameter is defined as $\sigma=\frac{\delta_{32}+\delta_{43}}{\psi_{32}-\psi_{43}}$

The dependence of this parameter on $H_{43}^{\text {exp }}$ is shown in Fig. 16. Here $\sigma=1$ corresponds to $H_{43, \text { ovl }}^{\exp } \approx 7.6034 \cdot 10^{-7}$. Thus, the overlapping of the $(3,2)$ and $(4,3)$ modes occurs for $H_{43}^{\exp } \in\left(8 \cdot 10^{-7}, 10^{-6}\right)$ which are larger than $H_{43, \text { ovlp }}^{\exp }$.

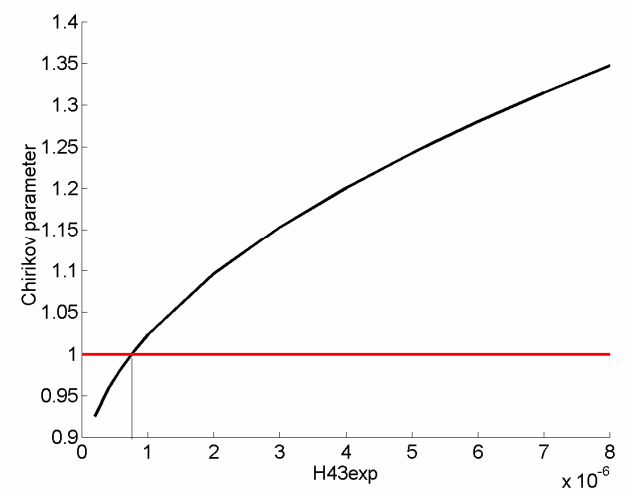

Figure 16. The Chirikov parameter as a function of $H_{43}^{\exp }$. 
In the experiment $\sigma \approx 1.2578>>1$. This corresponds to a pronounced overlapping of the $(3,2)$ and $(4,3)$ modes which is in agreement with the phase portrait shown in Fig. 14. If $H_{43}^{\exp }$ is small, the perturbation acts below the stochastic zone and the barriers are rapidly destroyed. When $H_{43}^{\text {exp }}$ becomes large enough, the perturbation acts mainly inside the stochastic zone and the external barriers are slowly destroyed affecting transport. This explains the different shapes of evolution of the stochastic area for $H_{43}^{\exp }<10^{-6}$ and $H_{43}^{\exp }>10^{-6}$ seen in Fig. 15. The next sudden enlargement occurs when the $(5,4)$ mode is stochastized for $H_{43}^{\text {exp }}>5.4 \cdot 10^{-6}$. This is shown in Fig. 17 where the trajectory starting at $(\pi / 3,0.175)$ is plotted. Here the stochastized $(7,5),(11,8)$ and $(4,3)$ modes can be easily identified.

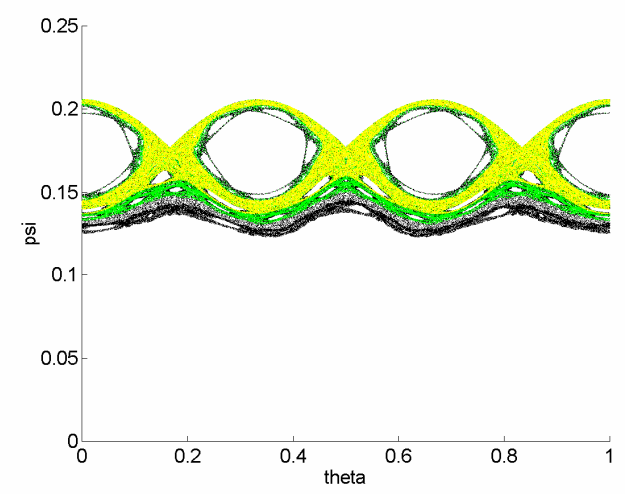

Figure 17. The trajectory starting from $(\pi / 3,0.175)$ in the case of the experimental perturbation of the $(3,2)$ mode, $H_{43}^{\text {exp }}=6 \cdot 10^{-7}$ (yellow), $H_{43}^{\text {exp }}=8 \cdot 10^{-7}$ (green) and $H_{43}^{\text {exp }}=10^{-6}$ (black).

A similar analysis can be carried out for the size of the area of the $(3,2)$ mode.

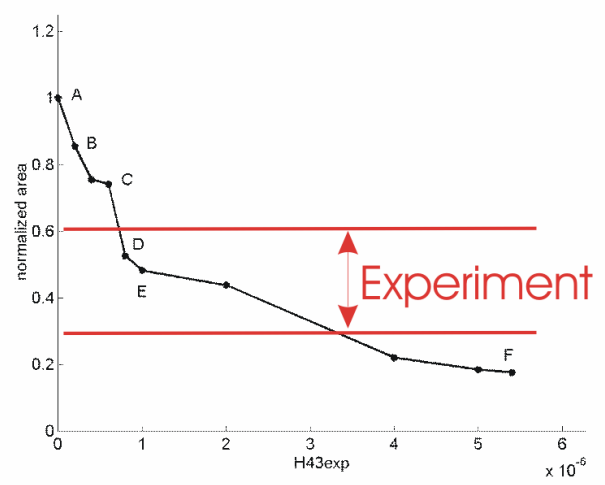

Figure 18. The area of the $(3,2)$ island as a function of $H_{43}^{\exp }$. Experiment means the relation between the island width before and after the $(3,2)$ amplitude drop obtained by means of the ECE and magnetic measurements. 
The most pronounced decrease of the area of the $(3,2)$ island is observed between the points $C$ and $D$, i.e. between $H_{43}^{\exp }=7.5 \cdot 10^{-7}$ and $H_{43}^{\exp }=5.5 \cdot 10^{-7}$. This can be explained by the fact that the secondary chain of five islands located inside the main island enters into the stochastic zone. The continuous decrease of the internal area seen in Fig. 18 is related to stochastization of other secondary thinner island chains. The next pronounced decrease of the area would appear when the secondary chain of four islands would become stochastic. This would happen for $H_{43}^{\exp }$ larger than the experimental value $5.4 \cdot 10^{-6}$. We can also estimate the island area reduction from the ECE and magnetic measurements. The relation between the measured island width after and before the amplitude drop corresponds to the experimental value of the island area reduction: $\max \left(w_{\text {after }}\right) / \max \left(w_{\text {before }}\right)=0.36-0.61$ (see Fig.18). This experimental estimation assumes the same shape of the island, $w(\theta)=w_{\max } \cdot \cos (m \cdot \theta)$, before and after the amplitude drop. It does not take into account the increase of the stochastic region at the X-point with increasing perturbations. For this reason the experimental values are somewhat larger than predicted by the coarse-grained method.

These studies prove that the experimental perturbation $H_{43}^{\exp }=5.4 \cdot 10^{-6}$ does not play any special role in the dynamics of the system. For somewhat smaller or larger perturbation amplitudes the dynamics and the main characteristics of the stochastic zone would be the same.

In order to describe the complicated dynamics inside the stochastic zones shown in Fig. 14, a single magnetic line is followed along a large number of iterations. Here it is assumed that $H_{43}^{\exp }=6 \cdot 10^{-7}$. The motion of the magnetic line can be partially understood by examining the values of the toroidal magnetic flux $\psi$ along the trajectory. The dependence of $\psi$ on time (number of iterations) is shown in Fig. 19. Different patterns of the motion can be distinguished:

- a wandering in the entire stochastic zone can be observed when $\psi$ varies in the range of all possible values. The zones with this property are marked by $a$.

- a pseudo-trapping or temporary confinement in the inner shell below $\psi=0.17$. These zones are marked by $b$.

- a deep sticking around some island chains. These zones are marked by $c$. 


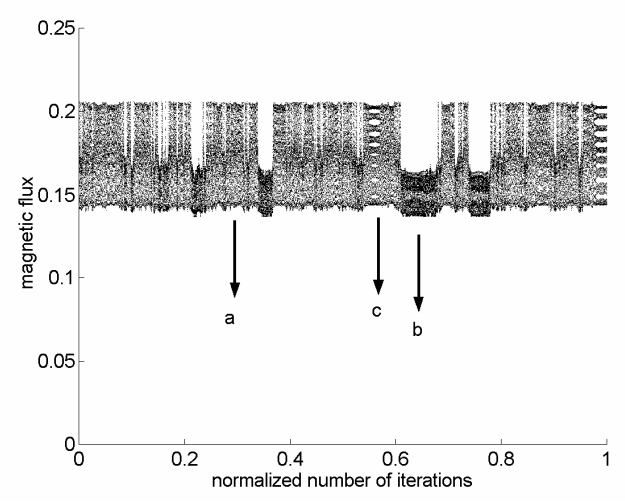

Figure 19. Time evolution of the toroidal magnetic flux along a stochastic trajectory in the case of $H_{43}^{\text {exp }}=6 \cdot 10^{-7}$.

At the beginning the magnetic line wanders in the whole stochastic zone. After this, it stays for some time bellow the $(3,2)$ mode, because it can not traverse the remnant of the KAM barrier which separates the $(3,2)$ and $(7,5)$ modes for weak perturbations. The stages $a, b$, and $c$ are of various lengths and they appear in an irregular manner. Hence the dynamics of the magnetic line can be interpreted as a stochastic one. Such an intermittent behaviour generates the strong increase of transport, phenomenon that was already observed in studying other systems which model the magnetic field in tokamaks [8].

Another trajectory starting from the stochastic zone would exhibit a different distribution and different lengths of the stages $a, b$, and $c$, but the trajectory would wander inside the same stochastic region.

\section{Summary and discussion}

In this paper we have applied the mapping method for the field line tracing to investigate the role of stochastization in the FIR and during minor disruption in the ASDEX Upgrade tokamak. Using the magnetic and ECE measurements we have reconstructed the safety factor profile and perturbations due to the MHD modes and implemented them into the Hamiltonian formalism of the field line tracing. As was pointed out, it is very difficult to determine accurately these quantities from the experiment. Moreover, the perturbation amplitudes differ by a factor two for different discharges and for different time periods of the same discharge. In spite of these difficulties our analysis of the FIR-NTMs strongly suggests that the nonlinear interaction between the $(3,2)$ and $(4,3)$ modes leads to stochastization and that the presence of the $(4,3)$ mode really is able to prevent the $(3,2)$ mode from growing to its saturated island size. Here the $(1,1)$ mode, which is needed for a nonlinear coupling between the modes, has a negligible influence on stochastization itself. Similar conclusions refer to the interaction between the $(4,3)$ and $(5,4)$ modes. Variations of the perturbed fluxes and safety factor profile inside the error bars do not change this result. We also have found that stochastization explains some cases of minor disruption observed in the ASDEX Upgrade tokamak. In these cases the interaction of the $(3,1)$ and $(2,1)$ tearing modes create stochastic 
region between the resonant surfaces. The temperature profile becomes flat in this region, which leads to temporary deterioration of the plasma confinement. The two cases considered in the paper show that stochastization can be extremely important in the interaction of several MHD modes. Here stochastization plays a major role in the plasma transport.

In this paper we also have tested two criteria of quantitative description of stochastization. In the future, we plan to convert these criteria into the diffusion coefficient and use it in non-linear MHD simulations. As a next step we will also extend our calculations to the framework of a real ASDEX Upgrade geometry.

\section{Acknowledgments}

The authors are grateful to V. Kuzovkov for useful remarks related to quantitative aspects of stochaticity.

\section{References}

[1] S. Günter, A. Gude, M. Maraschek, S. Sesnic, H. Zohm, ASDEX Upgrade team, and D. Howell, Phys. Rev. Lett. 87, 275001 (2001).

[2] A. Gude, S. Günter, M. Maraschek, H. Zohm, and ASDEX Upgrade team, Nucl. Fusion 42, 833 (2002)

[3] S. Günter, M. Maraschek, M. de Baar, D. F. Howell, E. Poli, E. Strumberger, C. Tichmann, ASDEX Upgrade team, and Contributors to the EFDA-JET Workprogramme, Nucl. Fusion, 44, 524 (2004).

[4] M. Maraschek, G. Gantenbein, T. P. Goodman, S. Günter, D.F. Howell, F. Leuterer, A. Mück, O. Sauter,

H. Zohm, and ASDEX Upgrade team, Nucl. Fusion 45, 1369 (2005).

[5] G. Gantenbein, A. Keller, M. Maraschek, W. Suttrop, H. Zohm, and ASDEX Upgrade team, 30th EPS Conf. (St.Peterburg) vol 27A, p P1.187.

[6] W. Suttrop, K. Büchl, J. C. Fuchs, M. Kaufmann, K. Lackner, M. Maraschek, V. Mertens, R. Neu, M. Schittenhelm, M. Sokoll, H. Zohm, and ASDEX Upgrade team, Nucl. Fusion 37, 119 (1997).

[7] O. Dumbrajs, V. Igochine, D. Constantinescu, H. Zohm, Phys. Plasmas 12, 110704 (2005).

[8] J.H. Misguish, J.-D. Reuss, D. Constantinescu, G. Steinbrecher, M. Vlad, F. Spineanu, B. Weyssow, and R. Balescu., Ann. Phys. Fr. $28 \mathrm{~N}^{\circ} 6$ (2003).

[9] S.S. Abdullaev, Nucl. Fusion 44, S12 (2004).

[10] A. Punjabi, A.Verma and A. Boozer, Phys.Rev.Lett. 69, 3322 (1992).

[11] J.R.Cary and R.G. Littlejohn, Annals of Physics 151, 1 (1983).

[12] S.S. Abdullaev, K.H. Finken, A. Kaleck, and K.H. Spatschek., Phys. Plasmas 5196 (1998).

[13]M. Schittenhelm, H. Zohm and the ASDEX Upgrade Team, Nucl. Fusion 37, 1255 (1997).

[14] S.S. Abdullaev (private communication, 2005, Institut für Plasmaphysik, Forschungszentrum Jülich).

[15] V. Igochine, S. Guenter, M. Maraschek and the ASDEX Upgrade Team., Nucl. Fusion 43, 1801

(2003). 
[16] J.P. Meskat, H. Zohm, G. Gantenbein, S. Günter, M. Maraschek, W. Suttrop, Q. Yu and ASDEX Upgrade Team, Plasma Phys. Control. Fusion 43, 1325 (2001).

[17] S. Migliuolo, Nucl. Fusion 33, 1721 (1993).

[18] E. Ott, Chaos in dynamical systems, Cambridge University Press, 1993.

[19] R. S. MacKay, Renormalization in area-preserving maps, World Scientific Publishing Co., 1993., Singapore.

[20] T. M. Janaki, G. Rangarajan, S. Habib, and R. D. Ryne, Phys. Rev. E 60, 6614 (1999).

[21] J. Farey, London, Edinburgh and Dublin Phil. Mag., 385 (1816).

[22] B. V. Chirikov, At. Energy 6, 630 (1959) [Engl. Transl. J. Nucl. Energy, part C Plasma Phys 1, 253 (1960). 